\title{
REZENSIONEN
}

\section{Ohne Moos nix los. Neuere Publikationen zu den bundesstaatlichen Finanzbeziehungen}

Junkernheinrich, Martin, Stefan Korioth, Thomas Lenk, Henrik Scheller und Matthias Woisin (Hrsg.): Jahrbuch für öffentliche Finanzen 2010 (Schriften zur öffentlichen Verwaltung und öffentlichen Wirtschaft, Band 218), Berliner Wissenschafts-Verlag, Berlin 2010, 386 Seiten, $€ 59$,-.

Kastrop, Christian, Gisela Meister-Scheufelen und Margaretha Sudhof (Hrsg.): Die neuen Schuldenregeln im Grundgesetz. Zur Fortentwicklung der bundesstaatlichen Finanzbeziehungen (Schriften zur öffentlichen Verwaltung und öffentlichen Wirtschaft, Band 219), Berliner Wissenschafts-Verlag, Berlin 2010, 478 Seiten, € 59,-.

Buscher, Daniel: Der Bundesstaat in Zeiten der Finanzkrise. Ein Beitrag zur Reform der deutschen Finanz- und Haushaltsordnung (Föderalismusreform) (Schriften zum Öffentlichen Recht, Band 1158), Duncker \& Humblot, Berlin 2010, 533 Seiten, € 96,-.

Heise, Axel: Bündische Solidarität oder föderaler Wettbewerb? Finanzföderalismus in Deutschland, der Schweiz und den USA im Vergleich (Schriftenreihe des Europäischen Zentrums für Föderalismus-Forschung, Band 37), Nomos Verlagsgesellschaft, Baden-Baden 2010, 200 Seiten, $€ 39$,-

Die „Spielregeln“ der Finanzpolitik wurden im Jahre 2009 durch die Einführung der „Schuldenbremse“ in das Grundgesetz gravierend verändert. Die Möglichkeiten von Bund und Ländern, sich am Kapitalmarkt zu verschulden, sind damit erheblich eingeschränkt worden. Auch wenn die Schuldenbremse ihre volle Wirkung erst in einigen Jahren entfalten wird, beeinflusst sie bereits heute das finanzpolitische Handeln. Zudem steht die Neuordnung des gesamten Systems der Bund-Länder-Finanzbeziehungen auf der Tagesordnung, denn die gegenwärtigen Regelungen enden mit dem Jahr 2019. Vor diesem Hintergrund ist es nicht verwunderlich, dass Fragen der föderalen Finanzordnung derzeit vermehrt wissenschaftliche Aufmerksamkeit gewinnen.

Das „Jahrbuch für öffentliche Finanzen 2010“, nun im zweiten Jahrgang vorgelegt, wurde auf Initiative von mehreren „Praktikern“ der öffentlichen Verwaltung und Wissenschaftlern gegründet, die sich mit den Fragen der öffentlichen Finanzen beschäftigen. Das konzeptionelle Ziel des Jahrbuches ist das regelmäßige Bereitstellen eines Länderfinanzberichtes und die Veröffentlichung von fachwissenschaftlichen Beiträgen, insbesondere aus der Sicht der Landespolitik.

Der Länderfinanzbericht, der etwa die Hälfte des Bandes ausmacht, schließt eine wichtige Lücke. Der Bundesfinanzminister legt jährlich seinen Bundesfinanzbericht vor, der Deutsche Städtetag veröffentlicht ebenfalls jährlich einen Gemeindefinanzbericht. Etwas Vergleichbares für die Länder gab es bisher nicht. Hierin liegt ein hoch zu veranschlagendes Verdienst der Herausgeber. In den Länderfinanzberichten finden sich Berichte zur Haushalts- und Finanzsituation jedes einzelnen Bundeslandes, die von Fachexperten anderer Länder (keine Eigenberichte!) verfasst werden. 
In den weiteren Beiträgen des zweiten Teils des Jahrbuches 2010 geht es um die öffentlichen Haushalte als Spiegel der Wirtschafts- und Finanzkrise, um die Kommunalfinanzen im Ländervergleich, die Sondervermögen von Bund und Ländern, den Bundesrechnungshof, die Umsetzung der Schuldenbremse in den Ländern, die Umsetzung der Kinderfördergesetze und die Versicherungsfunktion öffentlicher Haushalte. Der Fokus des Jahrbuches richtet sich damit - entsprechend dem Modell des deutschen Exekutivföderalismus - eher auf die Implementation von Politik, weniger auf die regulative Politik. Bekanntlich stiftet, wer etwas zum zweiten Mal tut, eine Tradition. Es ist zu hoffen und zu wünschen, dass mit dem zweiten Jahrgang des Jahrbuches für öffentliche Finanzen eine solche Tradition begründet wurde.

Einem Spezialproblem des Jahrbuches für öffentliche Finanzen 2010 - der Schuldenbremse - widmet sich der Band „Die neuen Schuldenregeln im Grundgesetz“. Auch dieser ist in erster Linie aus der Innenperspektive, nämlich von Praktikern der Politik, geschrieben.

Im ersten Teil wird das Schuldenregime für den Bund mit dem Schwerpunkt auf der Zeit nach 1969 und den neueren konzeptionellen Arbeiten des Bundesfinanzministeriums erläutert. Daran schließt sich eine Analyse der politischen Prozesse in der Föderalismuskommission II und eine Darstellung der neuen Regeln aus der Sicht des Bundes an. In einem weiteren Schritt werden die Bund-Länder-Finanzfragen und die Umsetzung der Schuldenbremse in den Ländern thematisiert. Der dritte Abschnitt wendet sich den weiteren Perspektiven zu, innerstaatlich geht es unter anderem um den Finanzausgleich und die finanziellen Aspekte der Wirtschafts- und Währungsunion sowie die G-20-Perspektive.

In den ersten gut 200 Seiten dominiert die Innensicht der an diesen Prozessen beteiligten Personen. Die folgenden 250 Seiten hingegen sind aus der Außenperspektive geschrieben worden: Externe Autoren und Vertreter der Wissenschaft diskutieren die Bedeutung der deutschen Schuldenbremse im Hinblick auf die Wirtschafts- und Währungsunion, vergleichen sie mit anderen Fiskalregimen und fragen nach den Erfolgsaussichten. Abschließende Bewertungen gehen auf Anreizprobleme, die Justitiabilität, Schwierigkeiten der Prognostik und die besonderen Probleme des Bund-Länder-Verhältnisses ein.

Der Band ist eine willkommene Ergänzung der vom Deutschen Bundestag und Bundesrat herausgegebenen Materialsammlung zur zweiten Föderalismuskommission. Während es konkret um die Intentionen und Arbeiten der Föderalismuskommission II geht, werden außerdem ungewöhnlich gute Einblicke geboten in die Problematik der Staatsverschuldung und die Strategie, sie beherrschbar zu machen. Besonders lesenswert sind die Einsichten der externen Autoren.

Bei der von Daniel Buscher vorgelegten Monografie „Der Bundesstaat in Zeiten der Finanzkrise" handelt es sich um eine von der Universität Bremen angenommene juristische Dissertation, die einen eher ambivalenten Eindruck hinterlässt. Einerseits beeindrucken die rechtshistorisch abgeleitete und systematisch angelegte Diskussion der deutschen Bundesstaatlichkeit und das detaillierte Eingehen auf die Finanzverfassung des Grundgesetzes. Hervorzuheben ist hier auch die Auseinandersetzung mit den fünf Urteilen des Bundesverfassungsgerichtes zu den Bund-Länder-Finanzbeziehungen. Andererseits wird dem informierten Leser - abgesehen von der allerjüngsten Entwicklung - wenig Neues geboten. Im Unterschied zu anderen zum Thema vorgelegten Arbeiten bleibt die Untersuchung weitestgehend rechtsimmanent. Finanzpolitische oder ökonomische Fragen oder politikwissenschaftliche Aspekte beispielsweise im Hinblick auf Handlungsrestriktionen durch den Par- 
teienwettbewerb findet der Leser nicht. Bedauerlich ist dies besonders, wenn es um Reformoptionen geht. Die Reformbedürftigkeit der Finanzverfassung wird nur aus der Verschuldung abgeleitet. Wirkliche oder vermeintliche Verwerfungen werden zwar behauptet, aber kaum belegt oder begründet (S. 212 ff.). Dies gilt auch für die „Vorgaben für künftige Reformen der Finanzordnung". Dabei unterbleibt leider die Befassung mit zentralen Fragen der bis 2019 anstehenden Reform des Finanzausgleichs, etwa die Berücksichtigung des demografischen Wandels oder die ungleichmäßig streuenden Belastungen der Länder durch die Bundespolitik. Stattdessen wird zum wiederholten Male die Debatte über eine Länderneugliederung geführt. So kann Buschers Arbeit nicht die Standardwerke ersetzen.

Der Titel der von Axel Heise vorgelegten Untersuchung führt in die Irre: Angekündigt wird eine vergleichende Analyse der bundesstaatlichen Finanzbeziehungen, geliefert wird eine tour d'horizon verschiedener Aspekte bundesstaatlicher Ordnungen, die alle irgendwie mit Finanzen zusammenhängen. Auf 18 Seiten wird das Thema Föderalismus und Finanzordnung abgehandelt; auf 14 Seiten erfährt der Leser etwas über die Entwicklungsphasen von Föderalismus und Finanzbeziehungen; der föderalen Struktur und den Finanzbeziehungen werden immerhin 27 Seiten gewidmet, wobei unter "Struktur" so disparate Aspekte wie föderale Beziehungen, Regierungssystem, Parteiensystem, Politische Kultur, Steuererhebung und Steuereinnahmen, Solidarität im Bundesstaat und „die Ausgabenseite“ subsumiert werden. Schließlich geht Heise auf knapp 20 Seiten anhand von sechs Beispielen der Frage nach, warum es in den drei genannten Staaten Aufsteiger- und Absteigerregionen gibt. Im Ergebnis stellt er fest, dass die Frage nach den Gründen für Erfolg oder Misserfolg einer Region nicht eindeutig beantwortet werden kann.

Auffällig, teilweise unerfreulich ist, dass der Verfasser Analyse und Wertung nicht hinreichend trennt. Gleich zu Beginn der Arbeit (S. 11) werden die gängigen kritischen Schlagworte aneinandergereiht, der „Spiegel“ zitiert und diese Fundstücke als Grund für die beiden Föderalismuskommissionen ausgegeben. Flapsige Formulierungen, in denen den Ministerpräsidenten „Angst vor der eigenen Courage“ (S. 12) vorgehalten wird, stärken nicht die wissenschaftliche Solidität. Kontroverse Meinungen und Positionen im wissenschaftlichen Diskurs werden arbiträr verwandt und als gesichert dargestellt. Insgesamt bleibt der Erkenntnisgewinn gering. Eigentlich schade, denn die im zweiten Teil aufgeworfene, aber unzureichend behandelte Frage nach den Ursachen von Erfolg und Misserfolg von Regionen ist wichtig.

Wolfgang Renzsch

Föderalismus in Deutschland und der Welt: gelungener Überblick

Sturm, Roland: Föderalismus. Eine Einführung, 2. Auflage, Nomos Verlagsgesellschaft, BadenBaden 2010, 242 Seiten, $€ 19,90$.

Obwohl der Föderalismus nicht nur in Deutschland als Strukturprinzip der politischen Ordnung eine lange Tradition hat, sondern auch weitere Staaten Europas Reformen in Richtung eines föderalen, jedenfalls aber dezentralen Systems unternehmen (Italien, Großbritannien) beziehungsweise unternommen haben (Belgien, Spanien), sind Überblickswer- 